\title{
ON LINEARLY COUPLED SCHRÖDINGER SYSTEMS
}

\author{
ZHIJIE CHEN AND WENMING ZOU \\ (Communicated by Chuu-Lian Terng)
}

ABStRACT. We study the following system of nonlinear Schrödinger equations:

$$
\begin{cases}-\Delta u+u=f(u)+\lambda v, & x \in \mathbb{R}^{N}, \\ -\Delta v+v=g(v)+\lambda u, & x \in \mathbb{R}^{N} .\end{cases}
$$

Under almost optimal assumptions on $f$ and $g$, for small $\lambda>0$, we obtain positive radial solutions and study their asymptotic behaviors as $\lambda \rightarrow 0$.

\section{INTRODUCTION}

We consider the following system of nonlinear Schrödinger equations:

$$
\left\{\begin{array}{l}
-\Delta u+u=f(u)+\lambda v, \quad x \in \mathbb{R}^{N}, \\
-\Delta v+v=g(v)+\lambda u, \quad x \in \mathbb{R}^{N}, \\
u(x), v(x) \rightarrow 0, \quad \text { as }|x| \rightarrow+\infty,
\end{array}\right.
$$

where $\lambda \geq 0, N \geq 3, f$ and $g$ are Carathéodory functions. Systems of this type arise in nonlinear optics (cf. [1]). It is well known that a solution $(u, v) \in H^{1}\left(\mathbb{R}^{N}\right) \times$ $H^{1}\left(\mathbb{R}^{N}\right)$ of (1.1) is called a bound state. A bound state such that $(u, v) \neq(0,0)$, respectively $u>0, v>0$, is called a nontrivial, respectively positive, bound state. A solution is called a ground state if $(u, v) \neq(0,0)$ and its energy is minimal among the energy of all the nontrivial bound states of (1.1). A ground state satisfying $u>0$ and $v>0$ is called a positive ground state. Obviously, the bound states of (1.1) are the critical points of the functional $I_{\lambda}: H^{1}\left(\mathbb{R}^{N}\right) \times H^{1}\left(\mathbb{R}^{N}\right) \rightarrow \mathbb{R}$ given by

$$
\begin{aligned}
I_{\lambda}(u, v):= & \frac{1}{2} \int_{\mathbb{R}^{N}}\left(|\nabla u|^{2}+|u|^{2}\right) d x+\frac{1}{2} \int_{\mathbb{R}^{N}}\left(|\nabla v|^{2}+|v|^{2}\right) d x \\
& -\int_{\mathbb{R}^{N}}(F(u)+G(v)) d x-\lambda \int_{\mathbb{R}^{N}} u v d x
\end{aligned}
$$

if $I_{\lambda} \in C^{1}\left(H^{1}\left(\mathbb{R}^{N}\right) \times H^{1}\left(\mathbb{R}^{N}\right), \mathbb{R}\right)$. Here $F(u):=\int_{0}^{u} f(s) d s$ and $G(v):=\int_{0}^{v} g(s) d s$. Let $2^{*}=\frac{2 N}{N-2}$. Assume that

$\left(\mathbf{F}_{\mathbf{1}}\right) \quad f, g \in C(\mathbb{R}, \mathbb{R})$, and $\lim _{s \rightarrow 0} \frac{f(s)}{s}=\lim _{s \rightarrow 0} \frac{g(s)}{s}=0$.

$\left(\mathbf{F}_{\mathbf{2}}\right)$ There exists $p \in\left(1,2^{*}-1\right)$ such that

$$
\limsup _{s \rightarrow+\infty} \frac{f(s)}{s^{p}}<+\infty \text { and } \limsup _{s \rightarrow+\infty} \frac{g(s)}{s^{p}}<+\infty .
$$

Received by the editors March 13, 2012.

2010 Mathematics Subject Classification. Primary 35B40, 35B45.

This work was supported by NSFC (11025106, 11271386, 10871109). 
$\left(\mathbf{F}_{3}\right)$

$$
\sup _{s>0} \frac{2 F(s)}{s^{2}}>1 \text { and } \sup _{s>0} \frac{2 G(s)}{s^{2}}>1 .
$$

Here we are concerned with positive solutions of (1.1), and in the sequel we assume without loss of generality that

$$
f(s) \equiv g(s) \equiv 0 \quad \text { for all } s \leq 0 .
$$

In the scalar case, assumptions $\left(F_{1}\right)-\left(F_{3}\right)$ are called Berestycki-Lions conditions, which were introduced by Berestycki and Lions 7 to get a ground state solution for

$$
-\Delta u+u=f(u), \quad u \in H^{1}\left(\mathbb{R}^{N}\right) .
$$

Other results about (1.4) can been seen in [5, 6, 11, 15] and the references therein. On the other hand, there have also been some results on the linearly coupled system (1.1) in the past several years. In the case of $N \leq 3, f(s)=g(s)=s^{3}$, Ambrosetti, Colorado and Ruiz 3 proved that (1.1) has multi-bump solitons for $\lambda>0$ small enough. When $f(u)$ and $g(v)$ are replaced by $f(x, u)=(1+c(x))|u|^{p-1} u$ and $g(x, v)=(1+d(x))|v|^{p-1} v$ respectively, system (1.1) has been studied by Ambrosetti [2] with dimension $N=1$ and Ambrosetti, Cerami and Ruiz [4] with dimension $N \geq 2$. Brezis and Lieb [8] consider some systems of equations

$$
-\Delta u_{i}(x)=g^{i}(u(x)), i=1, \cdots, n,
$$

where the $n$ functions $g^{i}: \mathbb{R}^{n} \rightarrow \mathbb{R}$ are the gradients of some function $G \in C^{1}\left(\mathbb{R}^{n}\right)$, namely $g^{i}(u)=\partial G(u) / \partial u_{i}$. Under some conditions on $g^{i}$ (see (2.2), (2.3), (2.4) and (2.8) in [8), they proved that (1.5) has a ground state solution which belongs to $H^{1}\left(\mathbb{R}^{N}\right) \cap W_{\text {loc }}^{2, q}\left(\mathbb{R}^{N}\right)$ for any $q<+\infty$ (see Theorem 2.2 and Theorem 2.3 in [8]). Recently, Byeon, Jeanjean and Maris [10] proved that ground state solutions of (1.5) obtained in [8] must be radial up to a translation. Note that (1.1) is a special case of (1.5), and $\left(F_{1}\right)-\left(F_{3}\right)$ with $\lambda \in[0,1)$ are consistent with those conditions on $g^{i}$ in $[8$; it follows that (1.1) produces a ground state solution $u, v \in H^{1}\left(\mathbb{R}^{N}\right) \cap W_{\text {loc }}^{2, q}\left(\mathbb{R}^{N}\right)$ for $\lambda \in[0,1)$. Then some further properties of the ground states for (1.1) were studied by the authors in 12 . In particular, we showed the following result.

Theorem A (See [12, Theorem 1.3]). Suppose $N \geq 3$ and $f, g$ satisfy assumptions $\left(F_{1}\right)-\left(F_{3}\right)$. Then for $\lambda \in(0,1)$, (1.1) has a positive radially symmetric ground state $\left(u_{\lambda}, v_{\lambda}\right)$ such that $u_{\lambda}, v_{\lambda} \in C^{2}\left(\mathbb{R}^{N}\right)$.

Let $\lambda_{n} \in(0,1), n \in \mathbb{N}$, be any sequence with $\lambda_{n} \rightarrow 0$ as $n \rightarrow \infty$. Then, passing to a subsequence, $\left(u_{\lambda_{n}}, v_{\lambda_{n}}\right) \rightarrow(\hat{u}, \hat{v})$ strongly in $H^{1}\left(\mathbb{R}^{N}\right) \times H^{1}\left(\mathbb{R}^{N}\right)$ as $n \rightarrow \infty$, where one of the following conclusions holds:

(i) $\hat{u} \equiv 0$ and $\hat{v}$ is a positive radial ground state of

$$
-\Delta v+v=g(v), \quad v \in H^{1}\left(\mathbb{R}^{N}\right) .
$$

(ii) $\hat{v} \equiv 0$ and $\hat{u}$ is a positive radial ground state of (1.4).

The main result of the current paper is the following.

Theorem 1.1. Suppose $N \geq 3$ and $f, g$ satisfy assumptions $\left(F_{1}\right)-\left(F_{3}\right)$. Then there exists $\lambda_{0} \in(0,1)$ such that for any $0<\lambda<\lambda_{0}$, (1.1) has a positive radially symmetric bound state $\left(u_{\lambda}, v_{\lambda}\right) \in C^{2}\left(\mathbb{R}^{N}\right) \times C^{2}\left(\mathbb{R}^{N}\right)$.

Furthermore, let $\lambda_{n} \in\left(0, \lambda_{0}\right), n \in \mathbb{N}$, be any sequence with $\lambda_{n} \rightarrow 0$ as $n \rightarrow \infty$. Then, passing to a subsequence, $\left(u_{\lambda_{n}}, v_{\lambda_{n}}\right) \rightarrow(U, V)$ strongly in $H^{1}\left(\mathbb{R}^{N}\right) \times H^{1}\left(\mathbb{R}^{N}\right)$ 
as $n \rightarrow \infty$, where $U$ is a positive radial ground state of (1.4) and $V$ is a positive radial ground state of (1.6) respectively.

Remark 1.1. Obviously, for $\lambda>0$ sufficiently small, bound states that are obtained in Theorem 1.1 are different from ground states obtained in Theorem A. That is, (1.1) has at least two positive bound states for $\lambda>0$ sufficiently small.

Remark 1.2. Berestycki and Lions [7] showed that assumptions $\left(F_{1}\right)-\left(F_{3}\right)$ are almost optimal for the existence of ground states of (1.4) and (1.6). Therefore, assumptions $\left(F_{1}\right)-\left(F_{3}\right)$ in Theorem 1.1 are almost optimal.

The rest of this paper proves Theorem 1.1. Note that classical reduction approaches cannot be used because of the general nonlinearities $f$ and $g$. Here we will adopt the ideas of Byeon and Jeanjean [9]. In order to construct a spike solution of the singularly perturbed nonlinear elliptic problem

$$
-\varepsilon^{2} \Delta u+V(x) u=f(u), u \in H^{1}\left(\mathbb{R}^{N}\right),
$$

for $\varepsilon>0$ sufficiently small, Byeon and Jeanjean [9] developed a new variational approach. We will follow this variational approach to prove Theorem 1.1. However, this approach cannot be used directly, and we need some crucial modifications for our proof. Precisely, we will define a special mountain-pass value $c_{\lambda}$, where all paths are required to be bounded in $H^{1}\left(\mathbb{R}^{N}\right) \times H^{1}\left(\mathbb{R}^{N}\right)$ by the same constant which is independent of $\lambda$; see Section 2.

We give some notation here. Throughout this paper, we denote the norm of $L^{q}\left(\mathbb{R}^{N}\right)$ by $|u|_{q}=\left(\int_{\mathbb{R}^{N}}|u|^{q} d x\right)^{\frac{1}{q}}$ and positive constants (possibly different) by $C$. The standard scalar product and norm of $H^{1}\left(\mathbb{R}^{N}\right)$ are given by

$$
\langle u, v\rangle:=\int_{\mathbb{R}^{N}}(\nabla u \nabla v+u v) d x, \quad\|u\|^{2}:=\langle u, u\rangle .
$$

Define

$$
H_{r}^{1}\left(\mathbb{R}^{N}\right):=\left\{u \in H^{1}\left(\mathbb{R}^{N}\right): \quad u \text { is radially symmetric }\right\} .
$$

Denote $H:=H^{1}\left(\mathbb{R}^{N}\right) \times H^{1}\left(\mathbb{R}^{N}\right)$ with the norm $\|(u, v)\|^{2}:=\|u\|^{2}+\|v\|^{2}$, and denote $H_{r}:=H_{r}^{1}\left(\mathbb{R}^{N}\right) \times H_{r}^{1}\left(\mathbb{R}^{N}\right)$. In the sequel it is understood that $\lambda \in[0,1)$.

\section{Proof of Theorem 1.1}

In this section, we consider the functional $I_{\lambda}$ restricted to $H_{r}$. By Palais' Symmetric Criticality Principle, any critical points of $I_{\lambda}: H_{r} \rightarrow \mathbb{R}$ are radially symmetric bound states of (1.1). The energy functionals of (1.4) and (1.6) are given by

$$
\begin{aligned}
& J_{1}(u)=\frac{1}{2} \int_{\mathbb{R}^{N}}\left(|\nabla u|^{2}+u^{2}\right) d x-\int_{\mathbb{R}^{N}} F(u) d x, \quad u \in H^{1}\left(\mathbb{R}^{N}\right), \\
& J_{2}(v)=\frac{1}{2} \int_{\mathbb{R}^{N}}\left(|\nabla v|^{2}+v^{2}\right) d x-\int_{\mathbb{R}^{N}} G(v) d x, \quad v \in H^{1}\left(\mathbb{R}^{N}\right) .
\end{aligned}
$$

Under assumptions $\left(F_{1}\right)-\left(F_{3}\right)$, Berestycki and Lions [7] proved that (1.4) (resp. (1.6) ) has a positive ground state solution. They also showed that each solution $U$ of (1.4) (resp. $V$ of (1.6) ) satisfies Pohozaev's identity

$$
J_{3}(U):=\int_{\mathbb{R}^{N}}|\nabla U|^{2} d x+2^{*} \int_{\mathbb{R}^{N}} \frac{U^{2}}{2}-F(U) d x=0
$$




$$
\left(\text { resp. } \quad J_{4}(V):=\int_{\mathbb{R}^{N}}|\nabla V|^{2} d x+2^{*} \int_{\mathbb{R}^{N}} \frac{V^{2}}{2}-G(V) d x=0\right) .
$$

Later, Byeon, Jeanjean and Maris [10] proved that ground state solutions of (1.4) (resp. (1.6) ) must be radial up to a translation. By (1.3), any nontrivial solutions of (1.4) (resp. (1.6)) must be positive. Define

$$
\begin{aligned}
S_{1}:=\left\{U \in H_{r}^{1}\left(\mathbb{R}^{N}\right):\right. & U \text { is a positive ground state of (1.4) }\} \\
S_{2}:=\left\{V \in H_{r}^{1}\left(\mathbb{R}^{N}\right):\right. & V \text { is a positive ground state of (1.6) }\}, \\
& X:=S_{1} \times S_{2} .
\end{aligned}
$$

Take fixed $U_{0} \in S_{1}$ and $V_{0} \in S_{2}$, and denote the least energy of (1.4) and (1.6) respectively by

$$
M_{1}:=J_{1}\left(U_{0}\right) \text { and } M_{2}:=J_{2}\left(V_{0}\right) \text { respectively. }
$$

Then $M_{1}>0$ and $M_{2}>0$ (cf. [7]). Moreover, [14, Lemma 3.1] says that

$$
M_{1}=\inf _{\substack{u \in H^{1}\left(\mathbb{R}^{N}\right) \backslash\{0\} \\ J_{3}(u)=0}} J_{1}(u), \quad M_{2}=\inf _{\substack{v \in H^{1}\left(\mathbb{R}^{N}\right) \backslash\{0\} \\ J_{4}(v)=0}} J_{2}(v) .
$$

Without loss of generality, we assume that

$$
M_{1} \leq M_{2} .
$$

Lemma 2.1. $X$ is compact in $H_{r}$, and there exist constants $C_{2}>C_{1}>0$ such that

$$
C_{1} \leq\|U\|,\|V\| \leq C_{2}, \quad \forall(U, V) \in X .
$$

Proof. By [9, Proposition 1], we see that $S_{1}$ and $S_{2}$ are both compact in $H_{r}^{1}\left(\mathbb{R}^{N}\right)$. Since $M_{1}>0$ and $M_{2}>0$, Lemma 2.1 follows trivially. have

For $t, s>0$, we define $U_{0, t}(x):=U_{0}\left(\frac{x}{t}\right)$ and $V_{0, s}(x):=V_{0}\left(\frac{x}{s}\right)$. Then by (2.1) we

$$
\begin{aligned}
J_{1}\left(U_{0, t}\right) & =\int_{\mathbb{R}^{N}} \frac{t^{N-2}}{2}\left|\nabla U_{0}\right|^{2}+\frac{t^{N}}{2} U_{0}^{2}-t^{N} F\left(U_{0}\right) d x \\
& =\left(\frac{t^{N-2}}{2}-\frac{(N-2) t^{N}}{2 N}\right) \int_{\mathbb{R}^{N}}\left|\nabla U_{0}\right|^{2} d x .
\end{aligned}
$$

Note that

$$
J_{1}\left(U_{0,1}\right)=\max _{t>0} J_{1}\left(U_{0, t}\right)=J_{1}\left(U_{0}\right)=M_{1} .
$$

It is easily seen that there exists $0<t_{0}<1<t_{1}$ such that

$$
J_{1}\left(U_{0, t}\right) \leq M_{1} / 4 \text { for } t \in\left(0, t_{0}\right] \cup\left[t_{1}, \infty\right) .
$$

Similarly, there exists $0<s_{0}<1<s_{1}$ such that

$$
J_{2}\left(V_{0, s}\right) \leq M_{1} / 4 \text { for } s \in\left(0, s_{0}\right] \cup\left[s_{1}, \infty\right) .
$$

Define

$$
\begin{aligned}
\widetilde{\gamma}_{1}(t):= & U_{0, t} \text { for } 0<t \leq t_{1}, \quad \widetilde{\gamma}_{1}(0):=0 ; \\
\widetilde{\gamma}_{2}(s):= & V_{0, s} \text { for } 0<s \leq s_{1}, \quad \widetilde{\gamma}_{2}(0):=0 ; \\
& \widetilde{\gamma}(t, s):=\left(\widetilde{\gamma}_{1}(t), \widetilde{\gamma}_{2}(s)\right) .
\end{aligned}
$$


Then there exists a constant $\mathcal{C}>0$ such that

$$
\max _{(t, s) \in\left[0, t_{1}\right] \times\left[0, s_{1}\right]}\|\widetilde{\gamma}(t, s)\| \leq \mathcal{C} .
$$

Denote $Q:=\left[0, t_{1}\right] \times\left[0, s_{1}\right]$ for convenience. Recalling $C_{2}$ in Lemma 2.1, we define

$$
c_{\lambda}:=\inf _{\gamma \in \Gamma} \max _{(t, s) \in Q} I_{\lambda}(\gamma(t, s)), \quad d_{\lambda}:=\max _{(t, s) \in Q} I_{\lambda}(\widetilde{\gamma}(t, s)),
$$

where

$$
\begin{aligned}
\Gamma:=\left\{\gamma \in C\left(Q, H_{r}\right):\right. & \max _{(t, s) \in Q}\|\gamma(t, s)\| \leq 2 C_{2}+\mathcal{C}, \\
& \left.\gamma(t, s)=\widetilde{\gamma}(t, s) \quad \text { for }(t, s) \in Q \backslash\left(t_{0}, t_{1}\right) \times\left(s_{0}, s_{1}\right)\right\} .
\end{aligned}
$$

Lemma 2.2. $\lim _{\lambda \rightarrow 0} c_{\lambda}=\lim _{\lambda \rightarrow 0} d_{\lambda}=c_{0}=d_{0}=M_{1}+M_{2}$.

Proof. Recalling $\lambda \geq 0$ and the definition of $I_{\lambda}$ in (1.2), we have $I_{\lambda}(\widetilde{\gamma}(t, s)) \leq$ $I_{0}(\widetilde{\gamma}(t, s))$, and so

$$
\begin{aligned}
d_{\lambda} & \leq d_{0}=\max _{(t, s) \in Q} I_{0}(\widetilde{\gamma}(t, s))=\max _{t \in\left[0, t_{1}\right]} J_{1}\left(\widetilde{\gamma}_{1}(t)\right)+\max _{s \in\left[0, s_{1}\right]} J_{2}\left(\widetilde{\gamma}_{2}(s)\right) \\
& =J_{1}\left(\widetilde{\gamma}_{1}(1)\right)+J_{2}\left(\widetilde{\gamma}_{2}(1)\right)=J_{1}\left(U_{0}\right)+J_{2}\left(V_{0}\right)=M_{1}+M_{2} .
\end{aligned}
$$

Note that $\widetilde{\gamma} \in \Gamma$; we have $c_{\lambda} \leq d_{\lambda}$, that is,

$$
\limsup _{\lambda \rightarrow 0} c_{\lambda} \leq \liminf _{\lambda \rightarrow 0} d_{\lambda} \leq \limsup _{\lambda \rightarrow 0} d_{\lambda} \leq d_{0}, \quad c_{0} \leq d_{0} .
$$

On the other hand, for any $\gamma(t, s)=\left(\gamma_{1}(t, s), \gamma_{2}(t, s)\right) \in \Gamma$, we define $\Upsilon(\gamma):\left[t_{0}, t_{1}\right] \times$ $\left[s_{0}, s_{1}\right] \rightarrow \mathbb{R}^{2}$ by

$$
\Upsilon(\gamma)(t, s):=\left(J_{5}\left(\gamma_{1}(t, s)\right)-J_{6}\left(\gamma_{2}(t, s)\right), J_{5}\left(\gamma_{1}(t, s)\right)+J_{6}\left(\gamma_{2}(t, s)\right)-2\right),
$$

where $J_{5}, J_{6}: H^{1}\left(\mathbb{R}^{N}\right) \rightarrow \mathbb{R}$ are defined by

$$
J_{5}(u):=\left\{\begin{array}{ll}
\frac{2^{*} \int_{\mathbb{R} N} F(u)}{\int_{\mathbb{R}^{N}}|\nabla u|^{2}+\frac{2^{*}}{2} u^{2}}, & \text { if } u \neq 0, \\
0, & \text { if } u=0,
\end{array}, \quad J_{6}(u):= \begin{cases}\frac{2^{*} \int_{\mathbb{R} N} G(u)}{\int_{\mathbb{R}^{N}}|\nabla u|^{2}+\frac{2^{*}}{2} u^{2}}, & \text { if } u \neq 0, \\
0, & \text { if } u=0 .\end{cases}\right.
$$

By $\left(\mathbf{F}_{\mathbf{1}}\right)-\left(\mathbf{F}_{\mathbf{2}}\right)$ and the Sobolev inequality $|u|_{p+1}^{p+1} \leq C\|u\|^{p+1}$, it is easy to prove that $J_{5}, J_{6}$ are continuous. Similarly as in (2.5) it is easily seen that

$$
\begin{aligned}
\Upsilon(\widetilde{\gamma})(t, s)=\left(\frac{2^{*} t^{2} \int_{\mathbb{R}^{N}} F\left(U_{0}\right)}{\int_{\mathbb{R}^{N}}\left|\nabla U_{0}\right|^{2}+\frac{2^{*}}{2} t^{2} U_{0}^{2}}-\frac{2^{*} s^{2} \int_{\mathbb{R}^{N}} G\left(V_{0}\right)}{\int_{\mathbb{R}^{N}}\left|\nabla V_{0}\right|^{2}+\frac{2^{*}}{2} s^{2} V_{0}^{2}},\right. \\
\\
\left.\quad \frac{2^{*} t^{2} \int_{\mathbb{R}^{N}} F\left(U_{0}\right)}{\int_{\mathbb{R}^{N}}\left|\nabla U_{0}\right|^{2}+\frac{2^{*}}{2} t^{2} U_{0}^{2}}+\frac{2^{*} s^{2} \int_{\mathbb{R}^{N}} G\left(V_{0}\right)}{\int_{\mathbb{R}^{N}}\left|\nabla V_{0}\right|^{2}+\frac{2^{*}}{2} s^{2} V_{0}^{2}}-2\right) .
\end{aligned}
$$

Recalling (2.1)-(2.2) and $J_{3}\left(U_{0}\right)=J_{4}\left(V_{0}\right)=0$, we have $\Upsilon(\widetilde{\gamma})(1,1)=(0,0)$. By a direct computation, one gets that $\operatorname{deg}\left(\Upsilon(\widetilde{\gamma}),\left[t_{0}, t_{1}\right] \times\left[s_{0}, s_{1}\right],(0,0)\right)=1$. By (2.10) we see that for any $(t, s) \in \partial\left(\left[t_{0}, t_{1}\right] \times\left[s_{0}, s_{1}\right]\right), \Upsilon(\gamma)(t, s)=\Upsilon(\widetilde{\gamma})(t, s) \neq(0,0)$. Therefore, $\operatorname{deg}\left(\Upsilon(\gamma),\left[t_{0}, t_{1}\right] \times\left[s_{0}, s_{1}\right],(0,0)\right)$ is well defined and

$$
\operatorname{deg}\left(\Upsilon(\gamma),\left[t_{0}, t_{1}\right] \times\left[s_{0}, s_{1}\right],(0,0)\right)=\operatorname{deg}\left(\Upsilon(\widetilde{\gamma}),\left[t_{0}, t_{1}\right] \times\left[s_{0}, s_{1}\right],(0,0)\right)=1 .
$$


Then there exists $\left(t_{2}, s_{2}\right) \in\left[t_{0}, t_{1}\right] \times\left[s_{0}, s_{1}\right]$ such that $\Upsilon(\gamma)\left(t_{2}, s_{2}\right)=(0,0)$, that is, $J_{5}\left(\gamma_{1}\left(t_{2}, s_{2}\right)\right)=J_{6}\left(\gamma_{2}\left(t_{2}, s_{2}\right)\right)=1$. This implies $J_{3}\left(\gamma_{1}\left(t_{2}, s_{2}\right)\right)=J_{4}\left(\gamma_{2}\left(t_{2}, s_{2}\right)\right)=0$ and $\gamma_{i}\left(t_{2}, s_{2}\right) \neq 0$ for $i=1,2$. Combining these with (2.3), we have

$$
\begin{aligned}
\max _{(t, s) \in Q} I_{0}(\gamma(t, s)) & \geq I_{0}\left(\gamma\left(t_{2}, s_{2}\right)\right)=J_{1}\left(\gamma_{1}\left(t_{2}, s_{2}\right)\right)+J_{2}\left(\gamma_{2}\left(t_{2}, s_{2}\right)\right) \\
& \geq M_{1}+M_{2}=d_{0} .
\end{aligned}
$$

Therefore, $c_{0} \geq d_{0}$. By (2.12) one gets that $c_{0}=d_{0}$.

Assume by contradiction that $\liminf _{\lambda \rightarrow 0} c_{\lambda}<d_{0}$. Then there exists $\varepsilon>0$, $\lambda_{n} \rightarrow 0$ and $\gamma_{n}=\left(\gamma_{n, 1}, \gamma_{n, 2}\right) \in \Gamma$ such that

$$
\max _{(t, s) \in Q} I_{\lambda_{n}}\left(\gamma_{n}(t, s)\right) \leq d_{0}-2 \varepsilon .
$$

By the definition of $\Gamma$ in (2.10), there exists $n_{0}$ large enough such that

$$
\max _{(t, s) \in Q} \lambda_{n}\left|\int_{\mathbb{R}^{N}} \gamma_{n, 1}(t, s) \gamma_{n, 2}(t, s) d x\right| \leq C \lambda_{n} \leq \varepsilon, \quad \forall n \geq n_{0},
$$

and so

$$
\max _{(t, s) \in Q} I_{0}\left(\gamma_{n}(t, s)\right) \leq \max _{(t, s) \in Q} I_{\lambda_{n}}\left(\gamma_{n}(t, s)\right)+\varepsilon \leq d_{0}-\varepsilon, \quad \forall n \geq n_{0},
$$

a contradiction with (2.13). Therefore, $\liminf \operatorname{in}_{\lambda \rightarrow 0} c_{\lambda} \geq d_{0}$. Combining this with (2.12), we complete the proof.

Recalling that $X=S_{1} \times S_{2}$, we define

$$
X^{\delta}:=\left\{(u, v) \in H_{r}: \operatorname{dist}((u, v), X) \leq \delta\right\}, I_{\lambda}^{d}:=\left\{(u, v) \in H_{r}: I_{\lambda}(u, v) \leq d\right\} .
$$

Lemma 2.3. Let $C_{1}$ be as in Lemma 2.1. For a small $\delta \in\left(0, C_{1} / 2\right)$, there exist constants $0<\sigma<1$ and $\lambda_{1} \in(0,1)$ such that $\left\|I_{\lambda}^{\prime}(u, v)\right\| \geq \sigma$ for any $(u, v) \in$ $I_{\lambda}^{d_{\lambda}} \cap\left(X^{\delta} \backslash X^{\delta / 2}\right)$ and any $\lambda \in\left(0, \lambda_{1}\right)$.

Proof. Assume by contradiction that there exist $\lambda_{n} \rightarrow 0$ and $\left(u_{n}, v_{n}\right) \in I_{\lambda_{n}}^{d_{\lambda_{n}}} \cap$ $\left(X^{\delta} \backslash X^{\delta / 2}\right)$ such that $\left\|I_{\lambda_{n}}^{\prime}\left(u_{n}, v_{n}\right)\right\| \rightarrow 0$. By Lemma 2.1. $\left\{\left(u_{n}, v_{n}\right), n \geq 1\right\}$ are uniformly bounded in $H_{r}$. Recall that the Sobolev embedding $H_{r}^{1}\left(\mathbb{R}^{N}\right) \hookrightarrow L^{q}\left(\mathbb{R}^{N}\right)$ is compact for any $q \in\left(2,2^{*}\right)$. Up to a subsequence, we may assume that $\left(u_{n}, v_{n}\right) \rightarrow$ $(U, V)$ weakly in $H_{r}$ and strongly in $L^{q}\left(\mathbb{R}^{N}\right), q \in\left(2,2^{*}\right)$. Then $I_{0}^{\prime}(U, V)=0$; that is, $U$ (resp. $V$ ) is a solution of (1.4) (resp. (1.6)). Moreover, by $\left(F_{1}\right)-\left(F_{2}\right)$ we have

$$
\lim _{n \rightarrow \infty} \int_{\mathbb{R}^{N}} f\left(u_{n}\right) u_{n} d x=\int_{\mathbb{R}^{N}} f(U) U d x
$$

By $I_{\lambda_{n}}^{\prime}\left(u_{n}, v_{n}\right)\left(u_{n}, 0\right) \rightarrow 0$, we get that

$$
\left\|u_{n}\right\|^{2}=\int_{\mathbb{R}^{N}} f\left(u_{n}\right) u_{n} d x+o(1)=\int_{\mathbb{R}^{N}} f(U) U d x+o(1)=\|U\|^{2}+o(1),
$$

and so $u_{n} \rightarrow U$ strongly in $H_{r}^{1}\left(\mathbb{R}^{N}\right)$. Similarly, $v_{n} \rightarrow V$ strongly in $H_{r}^{1}\left(\mathbb{R}^{N}\right)$, and so $(U, V) \in X^{\delta}$, which implies that $U \not \equiv 0$ and $V \not \equiv 0$. By (1.3) and the strong maximum principle we have $U>0$ and $V>0$. Recalling Lemma 2.2 and the definition of $M_{1}, M_{2}$, we have

$$
\begin{aligned}
M_{1}+M_{2} & \leq J_{1}(U)+J_{2}(V)=I_{0}(U, V)=\lim _{n \rightarrow \infty} I_{\lambda_{n}}\left(u_{n}, v_{n}\right) \\
& \leq \lim _{n \rightarrow \infty} d_{\lambda_{n}}=M_{1}+M_{2} .
\end{aligned}
$$


This means $J_{1}(U)=M_{1}$ and $J_{2}(V)=M_{2}$, that is, $U \in S_{1}$ and $V \in S_{2}$. So $(U, V) \in X$, which contradicts $\left(u_{n}, v_{n}\right) \notin X^{\delta / 2}$ for any $n \geq 1$. This completes the proof.

From now on, we fix a small $\delta \in\left(0, \min \left\{\mathcal{C} / 2, C_{1} / 2\right\}\right)$ and corresponding $0<$ $\sigma<1$ and $\lambda_{1}>0$ such that conclusions in Lemma 2.3 hold.

Lemma 2.4. There exists $\lambda_{2} \in\left(0, \lambda_{1}\right]$ and $\alpha>0$ such that for any $\lambda \in\left(0, \lambda_{2}\right)$,

$$
I_{\lambda}(\widetilde{\gamma}(t, s)) \geq c_{\lambda}-\alpha \quad \text { implies that } \quad \widetilde{\gamma}(t, s) \in X^{\delta / 2} .
$$

Proof. Assume by contradiction that there exist $\lambda_{n} \rightarrow 0, \alpha_{n} \rightarrow 0$ and $\left(t_{n}, s_{n}\right) \in Q$ such that

$$
I_{\lambda_{n}}\left(\widetilde{\gamma}\left(t_{n}, s_{n}\right)\right) \geq c_{\lambda_{n}}-\alpha_{n} \text { and } \quad \widetilde{\gamma}\left(t_{n}, s_{n}\right) \notin X^{\delta / 2} .
$$

Passing to a subsequence, we may assume that $\left(t_{n}, s_{n}\right) \rightarrow(\tilde{t}, \tilde{s}) \in Q$. Then by Lemma 2.2 we have

$$
I_{0}(\tilde{\gamma}(\tilde{t}, \tilde{s})) \geq \lim _{n \rightarrow \infty} c_{\lambda_{n}}=M_{1}+M_{2} .
$$

Combining this with (2.5)-(2.6) and (2.11), it is easy to see that $(\tilde{t}, \tilde{s})=(1,1)$. Hence,

$$
\lim _{n \rightarrow \infty}\left\|\widetilde{\gamma}\left(t_{n}, s_{n}\right)-\widetilde{\gamma}(1,1)\right\|=0
$$

However, $\widetilde{\gamma}(1,1)=\left(U_{0}, V_{0}\right) \in X$, which is a contradiction to (2.14).

Let

$$
\alpha_{0}:=\min \left\{\frac{\alpha}{2}, \frac{M_{1}}{4}, \frac{1}{8} \delta \sigma^{2}\right\}
$$

where $\delta, \sigma$ are seen in Lemma 2.3. By Lemma 2.2 there exists $\lambda_{0} \in\left(0, \lambda_{2}\right]$ such that

$$
\left|c_{\lambda}-d_{\lambda}\right|<\alpha_{0}, \quad\left|c_{\lambda}-\left(M_{1}+M_{2}\right)\right|<\alpha_{0}, \quad \forall \lambda \in\left(0, \lambda_{0}\right) .
$$

Lemma 2.5. For fixed $\lambda \in\left(0, \lambda_{0}\right)$, there exists $\left\{\left(u_{n}, v_{n}\right)\right\}_{n=1}^{\infty} \subset X^{\delta} \cap I_{\lambda}^{d_{\lambda}}$ such that

$$
I_{\lambda}^{\prime}\left(u_{n}, v_{n}\right) \rightarrow 0 \quad \text { in } H_{r}, \quad \text { as } n \rightarrow \infty \text {. }
$$

Proof. Fix a $\lambda \in\left(0, \lambda_{0}\right)$. Assume by contradiction that there exists $0<l(\lambda)<1$ such that $\left\|I_{\lambda}^{\prime}(u, v)\right\| \geq l(\lambda)$ on $X^{\delta} \cap I_{\lambda}^{d_{\lambda}}$. Then there exists a pseudo-gradient vector field $T_{\lambda}$ in $H_{r}$ which is defined on a neighborhood $Z_{\lambda}$ of $X^{\delta} \cap I_{\lambda}^{d_{\lambda}}$ (cf. [16]) such that for any $(u, v) \in Z_{\lambda}$ there holds

$$
\begin{aligned}
\left\|T_{\lambda}(u, v)\right\| & \leq 2 \min \left\{1,\left\|I_{\lambda}^{\prime}(u, v)\right\|\right\}, \\
\left\langle I_{\lambda}^{\prime}(u, v), T_{\lambda}(u, v)\right\rangle & \geq \min \left\{1,\left\|I_{\lambda}^{\prime}(u, v)\right\|\right\}\left\|I_{\lambda}^{\prime}(u, v)\right\| .
\end{aligned}
$$

Let $\eta_{\lambda}$ be a Lipschitz continuous function on $H_{r}$ such that $0 \leq \eta_{\lambda} \leq 1, \eta_{\lambda} \equiv 1$ on $X^{\delta} \cap I_{\lambda}^{d_{\lambda}}$ and $\eta_{\lambda} \equiv 0$ on $H_{r} \backslash Z_{\lambda}$. Let $\xi_{\lambda}$ be a Lipschitz continuous function on $\mathbb{R}$ such that $0 \leq \xi_{\lambda} \leq 1, \xi_{\lambda}(l) \equiv 1$ if $\left|l-c_{\lambda}\right| \leq \frac{\alpha}{2}$ and $\xi_{\lambda}(l) \equiv 0$ if $\left|l-c_{\lambda}\right| \geq \alpha$. Let

$$
e_{\lambda}(u, v)=\left\{\begin{array}{lll}
-\eta_{\lambda}(u, v) \xi_{\lambda}\left(I_{\lambda}(u, v)\right) T_{\lambda}(u, v) & \text { if } \quad(u, v) \in Z_{\lambda}, \\
0 & \text { if } \quad(u, v) \in H_{r} \backslash Z_{\lambda} .
\end{array}\right.
$$

Then there exists a global solution $\psi_{\lambda}: H_{r} \times[0,+\infty) \rightarrow H_{r}$ for the initial value problem

$$
\left\{\begin{array}{l}
\frac{d}{d \theta} \psi_{\lambda}(u, v, \theta)=e_{\lambda}\left(\psi_{\lambda}(u, v, \theta)\right) \\
\psi_{\lambda}(u, v, 0)=(u, v)
\end{array}\right.
$$


It is easy to see that $\psi_{\lambda}$ has the following properties:

(1) $\psi_{\lambda}(u, v, \theta)=(u, v)$ if $\theta=0$ or $(u, v) \in H_{r} \backslash Z_{\lambda}$ or $\left|I_{\lambda}(u, v)-c_{\lambda}\right| \geq \alpha$.

(2) $\left\|\frac{d}{d \theta} \psi_{\lambda}(u, v, \theta)\right\| \leq 2$.

(3) $\frac{d}{d \theta} I_{\lambda}\left(\psi_{\lambda}(u, v, \theta)\right)=\left\langle I_{\lambda}^{\prime}\left(\psi_{\lambda}(u, v, \theta)\right), e_{\lambda}\left(\psi_{\lambda}(u, v, \theta)\right)\right\rangle \leq 0$.

Step 1. For any $(t, s) \in Q$, we claim that there exists $\theta_{t, s} \in[0,+\infty)$ such that $\psi_{\lambda}\left(\widetilde{\gamma}(t, s), \theta_{t, s}\right) \in I_{\lambda}^{c_{\lambda}-\alpha_{0}}$, where $\alpha_{0}$ is seen in (2.15).

Assume by contradiction that there exists $(t, s) \in Q$ such that

$$
I_{\lambda}\left(\psi_{\lambda}(\widetilde{\gamma}(t, s), \theta)\right)>c_{\lambda}-\alpha_{0},
$$

for any $\theta \geq 0$. Note that $\alpha_{0}<\alpha$; we see from Lemma 2.4 that $\widetilde{\gamma}(t, s) \in X^{\delta / 2}$. Note that $I_{\lambda}(\widetilde{\gamma}(t, s)) \leq d_{\lambda}<c_{\lambda}+\alpha_{0}$; we see from the property (3) that

$$
c_{\lambda}-\alpha_{0}<I_{\lambda}\left(\psi_{\lambda}(\widetilde{\gamma}(t, s), \theta)\right) \leq d_{\lambda}<c_{\lambda}+\alpha_{0}, \quad \forall \theta \geq 0 .
$$

This implies $\xi_{\lambda}\left(I_{\lambda}\left(\psi_{\lambda}(\widetilde{\gamma}(t, s), \theta)\right)\right) \equiv 1$. If $\psi_{\lambda}(\widetilde{\gamma}(t, s), \theta) \in X^{\delta}$ for all $\theta \geq 0$, then $\eta_{\lambda}\left(\psi_{\lambda}(\widetilde{\gamma}(t, s), \theta)\right) \equiv 1$, and $\left\|I_{\lambda}^{\prime}\left(\psi_{\lambda}(\widetilde{\gamma}(t, s), \theta)\right)\right\| \geq l(\lambda)$ for all $\theta>0$. Then

$$
I_{\lambda}\left(\psi_{\lambda}\left(\widetilde{\gamma}(t, s), \frac{\alpha}{l(\lambda)^{2}}\right)\right) \leq c_{\lambda}+\frac{\alpha}{2}-\int_{0}^{\frac{\alpha}{l(\lambda)^{2}}} l(\lambda)^{2} d t \leq c_{\lambda}-\frac{\alpha}{2},
$$

a contradiction. Thus, there exists $\theta_{t, s}>0$ such that $\psi_{\lambda}\left(\widetilde{\gamma}(t, s), \theta_{t, s}\right) \notin X^{\delta}$. Note that $\widetilde{\gamma}(t, s) \in X^{\delta / 2}$; there exist $0<\theta_{t, s}^{1}<\theta_{t, s}^{2} \leq \theta_{t, s}$ such that $\psi_{\lambda}\left(\widetilde{\gamma}(t, s), \theta_{t, s}^{1}\right) \in$ $\partial X^{\delta / 2}, \psi_{\lambda}\left(\widetilde{\gamma}(t, s), \theta_{t, s}^{2}\right) \in \partial X^{\delta}$ and $\psi_{\lambda}(\widetilde{\gamma}(t, s), \theta) \in X^{\delta} \backslash X^{\delta / 2}$ for all $\theta \in\left(\theta_{t, s}^{1}, \theta_{t, s}^{2}\right)$. Then by Lemma 2.3 we have $\left\|I_{\lambda}^{\prime}\left(\psi_{\lambda}(\widetilde{\gamma}(t, s), \theta)\right)\right\| \geq \sigma$ for all $\theta \in\left(\theta_{t, s}^{1}, \theta_{t, s}^{2}\right)$. Then using property (2) we have

$$
\delta / 2 \leq\left\|\psi_{\lambda}\left(\widetilde{\gamma}(t, s), \theta_{t, s}^{2}\right)-\psi_{\lambda}\left(\widetilde{\gamma}(t, s), \theta_{t, s}^{1}\right)\right\| \leq 2\left|\theta_{t, s}^{2}-\theta_{t, s}^{1}\right|,
$$

that is, $\theta_{t, s}^{2}-\theta_{t, s}^{1} \geq \delta / 4$. This implies

$$
\begin{aligned}
I_{\lambda}\left(\psi_{\lambda}\left(\widetilde{\gamma}(t, s), \theta_{t, s}^{2}\right)\right) & \leq I_{\lambda}\left(\psi_{\lambda}\left(\widetilde{\gamma}(t, s), \theta_{t, s}^{1}\right)\right)+\int_{\theta_{t, s}^{1}}^{\theta_{t, s}^{2}} \frac{d}{d \theta} I_{\lambda}\left(\psi_{\lambda}(u, v, \theta)\right) d \theta \\
& <c_{\lambda}+\alpha_{0}-\sigma^{2}\left(\theta_{t, s}^{2}-\theta_{t, s}^{1}\right) \leq c_{\lambda}+\alpha_{0}-\frac{1}{4} \delta \sigma^{2} \\
& \leq c_{\lambda}-\alpha_{0}
\end{aligned}
$$

which is a contradiction.

By Step 1 we can define $T(t, s):=\inf \left\{\theta \geq 0: I_{\lambda}\left(\psi_{\lambda}(\widetilde{\gamma}(t, s), \theta)\right) \leq c_{\lambda}-\alpha_{0}\right\}$ and let $\gamma(t, s):=\psi_{\lambda}(\widetilde{\gamma}(t, s), T(t, s))$. Then $I_{\lambda}(\gamma(t, s)) \leq c_{\lambda}-\alpha_{0}$ for all $(t, s) \in Q$.

Step 2. We shall prove that $\gamma(t, s) \in \Gamma$.

For any $(t, s) \in Q \backslash\left(t_{0}, t_{1}\right) \times\left(s_{0}, s_{1}\right)$, by (2.4), (2.6)-(2.8) and (2.15)-(2.16), we have

$$
\begin{aligned}
I_{\lambda}(\widetilde{\gamma}(t, s)) & \leq I_{0}(\widetilde{\gamma}(t, s))=J_{1}\left(\widetilde{\gamma}_{1}(t)\right)+J_{2}\left(\widetilde{\gamma}_{2}(s)\right) \\
& \leq M_{1} / 4+M_{2} \leq M_{1}+M_{2}-3 \alpha_{0}<c_{\lambda}-\alpha_{0},
\end{aligned}
$$

which implies that $T(t, s)=0$ and so $\gamma(t, s)=\widetilde{\gamma}(t, s)$.

From the definition of $\Gamma$ in (2.10), it suffices to prove that $\|\gamma(t, s)\| \leq 2 C_{2}+\mathcal{C}$ for all $(t, s) \in Q$ and $T(t, s)$ is continuous with respect to $(t, s)$. 
For any $(t, s) \in Q$, if $I_{\lambda}(\widetilde{\gamma}(t, s)) \leq c_{\lambda}-\alpha_{0}$, we have $T(t, s)=0$ and so $\gamma(t, s)=$ $\widetilde{\gamma}(t, s)$, and by (2.9) we see that $\|\gamma(t, s)\| \leq \mathcal{C}<2 C_{2}+\mathcal{C}$.

If $I_{\lambda}(\widetilde{\gamma}(t, s))>c_{\lambda}-\alpha_{0}$, then $\widetilde{\gamma}(t, s) \in X^{\delta / 2}$ and

$$
c_{\lambda}-\alpha_{0}<I_{\lambda}\left(\psi_{\lambda}(\widetilde{\gamma}(t, s), \theta)\right) \leq d_{\lambda}<c_{\lambda}+\alpha_{0}, \quad \forall \theta \in[0, T(t, s)) .
$$

This implies $\xi_{\lambda}\left(I_{\lambda}\left(\psi_{\lambda}(\widetilde{\gamma}(t, s), \theta)\right)\right) \equiv 1$ for $\theta \in[0, T(t, s))$. If $\psi_{\lambda}(\widetilde{\gamma}(t, s), T(t, s)) \notin$ $X^{\delta}$, then there exists $0<\theta_{t, s}^{1}<\theta_{t, s}^{2}<T(t, s)$ as above. Then we can prove that $I_{\lambda}\left(\psi_{\lambda}\left(\widetilde{\gamma}(t, s), \theta_{t, s}^{2}\right)\right) \leq c_{\lambda}-\alpha_{0}$ as above, which contradicts the definition of $T(t, s)$. Therefore, $\gamma(t, s)=\psi_{\lambda}(\widetilde{\gamma}(t, s), T(t, s)) \in X^{\delta}$. Then there exists $(u, v) \in X$ such that $\|\gamma(t, s)-(u, v)\| \leq \delta \leq \mathcal{C} / 2$. By Lemma 2.1 we have

$$
\|\gamma(t, s)\| \leq\|(u, v)\|+\mathcal{C} / 2 \leq 2 C_{2}+\mathcal{C} .
$$

To prove the continuity of $T(t, s)$, we fix any $(\tilde{t}, \tilde{s}) \in Q$. Assume that $I_{\lambda}(\gamma(\tilde{t}, \tilde{s}))<$ $c_{\lambda}-\alpha_{0}$ first. Then $T(\tilde{t}, \tilde{s})=0$ from the definition of $T(t, s)$. So $I_{\lambda}(\tilde{\gamma}(\tilde{t}, \tilde{s}))<c_{\lambda}-\alpha_{0}$. By the continuity of $\widetilde{\gamma}$, there exists $\tau>0$ such that for any $(t, s) \in(\tilde{t}-\tau, \tilde{t}+\tau) \times$ $(\tilde{s}-\tau, \tilde{s}+\tau) \cap Q$, we have $I_{\lambda}(\widetilde{\gamma}(t, s))<c_{\lambda}-\alpha_{0}$, that is, $T(t, s)=0$, and $T$ is continuous at $(\tilde{t}, \tilde{s})$. Now we assume that $I_{\lambda}(\gamma(\tilde{t}, \tilde{s}))=c_{\lambda}-\alpha_{0}$. Then from the previous proof we see that $\gamma(\tilde{t}, \tilde{s})=\psi_{\lambda}(\tilde{\gamma}(\tilde{t}, \tilde{s}), T(\tilde{t}, \tilde{s})) \in X^{\delta}$, and so

$$
\left\|I_{\lambda}^{\prime}\left(\psi_{\lambda}(\tilde{\gamma}(\tilde{t}, \tilde{s}), T(\tilde{t}, \tilde{s}))\right)\right\| \geq l(\lambda)>0 .
$$

Then for any $\omega>0$, we have $I_{\lambda}\left(\psi_{\lambda}(\tilde{\gamma}(\tilde{t}, \tilde{s}), T(\tilde{t}, \tilde{s})+\omega)\right)<c_{\lambda}-\alpha_{0}$. By the continuity of $\psi_{\lambda}$, there exists $\tau>0$ such that for any $(t, s) \in(\tilde{t}-\tau, \tilde{t}+\tau) \times(\tilde{s}-\tau, \tilde{s}+\tau) \cap Q$, we have $I_{\lambda}\left(\psi_{\lambda}(\widetilde{\gamma}(t, s), T(\tilde{t}, \tilde{s})+\omega)\right)<c_{\lambda}-\alpha_{0}$, so $T(t, s) \leq T(\tilde{t}, \tilde{s})+\omega$. It follows that

$$
0 \leq \limsup _{(t, s) \rightarrow(\tilde{t}, \tilde{s})} T(t, s) \leq T(\tilde{t}, \tilde{s}) .
$$

If $T(\tilde{t}, \tilde{s})=0$, we immediately have

$$
\lim _{(t, s) \rightarrow(\tilde{t}, \tilde{s})} T(t, s)=T(\tilde{t}, \tilde{s}) .
$$

If $T(\tilde{t}, \tilde{s})>0$, then for any $0<\omega<T(\tilde{t}, \tilde{s})$, similarly we have $I_{\lambda}\left(\psi_{\lambda}(\widetilde{\gamma}(\tilde{t}, \tilde{s}), T(\tilde{t}, \tilde{s})-\right.$ $\omega))>c_{\lambda}-\alpha_{0}$. By the continuity of $\psi_{\lambda}$ again, we see that

$$
\liminf _{(t, s) \rightarrow(\tilde{t}, \tilde{s})} T(t, s) \geq T(\tilde{t}, \tilde{s}) .
$$

So $T$ is continuous at $(\tilde{t}, \tilde{s})$. This completes the proof of Step 2 .

Now, we have proved that $\gamma(t, s) \in \Gamma$ and $\max _{(t, s) \in Q} I_{\lambda}(\gamma(t, s)) \leq c_{\lambda}-\alpha_{0}$, which contradicts the definition of $c_{\lambda}$. This completes the proof.

Lemma 2.6. Let $\lambda \in(0,1)$. Assume that $(u, v)$ is a nontrivial solution of (1.1); then $u>0$ and $v>0$.

Proof. It is easily seen from (1.1) that both $u \not \equiv 0$ and $v \not \equiv 0$. Assume by contradiction that $\left\{x \in \mathbb{R}^{N}: u(x)<0\right\}$ is not empty. By a standard regularity theory, we see that $u, v \in W_{\text {loc }}^{2, q}\left(\mathbb{R}^{N}\right)$ for any $q<+\infty$, which implies

$$
-\Delta u+u=f(u)+\lambda v, \quad \text { a.e. } x \in \mathbb{R}^{N},
$$

and $u, v \in C_{\text {loc }}^{1, \alpha}\left(\mathbb{R}^{N}\right), 0<\alpha<1$. Therefore, $\Delta u \in C\left(\mathbb{R}^{N}\right)$. 
Let $u\left(x_{1}\right)=\inf _{\mathbb{R}^{N}} u(x)<0$. We claim that $\Delta u\left(x_{1}\right) \geq 0$. Indeed, if $\Delta u\left(x_{1}\right)<0$, there exists $r>0$ such that

$$
u(x)<0, \quad \Delta u(x)<0, \quad \forall x \in B\left(x_{1}, r\right),
$$

which implies from the maximum principle (cf. [13]) that $\inf _{B\left(x_{1}, r\right)} u(x) \geq \inf _{\partial B\left(x_{1}, r\right)} u(x)$. Note that $u\left(x_{1}\right)=\inf _{\mathbb{R}^{N}} u(x)$; we obtain that $u \equiv$ const in $B(x, r)$ from the strong maximum principle (cf. [13]), so $\Delta u\left(x_{1}\right)=0$, which is a contradiction.

Thus $\Delta u\left(x_{1}\right) \geq 0$. Note that $f(s) \equiv 0$ for $s \leq 0$, and if we have $f\left(u\left(x_{1}\right)\right)=0$, then $-\Delta u\left(x_{1}\right)+u\left(x_{1}\right)=\lambda v\left(x_{1}\right) \leq u\left(x_{1}\right)<\lambda u\left(x_{1}\right)$, that is, $v\left(x_{1}\right)<\inf _{\mathbb{R}^{N}} u(x)<0$, so $\inf _{\mathbb{R}^{N}} v(x)<\inf _{\mathbb{R}^{N}} u(x)$. Similarly, we can obtain that $\inf _{\mathbb{R}^{N}} u(x)<\inf _{\mathbb{R}^{N}} v(x)$, which is a contradiction. Hence, $\left\{x \in \mathbb{R}^{N}: u(x)<0\right\}=\emptyset$, and $u \geq 0$. Similarly, $v \geq 0$. Then by the strong maximum principle (cf. [13]), we see that $u, v>0$.

Proof of Theorem 1.1. First we fix any $\lambda \in\left(0, \lambda_{0}\right)$. By Lemma 2.5 there exists $\left\{\left(u_{n}, v_{n}\right)\right\}_{n=1}^{\infty} \subset X^{\delta} \cap I_{\lambda}^{d_{\lambda}}$ such that

$$
I_{\lambda}^{\prime}\left(u_{n}, v_{n}\right) \rightarrow 0 \quad \text { in } H_{r}, \quad \text { as } n \rightarrow \infty .
$$

By Lemma 2.1, $\left\{\left(u_{n}, v_{n}\right), n \geq 1\right\}$ are uniformly bounded in $H_{r}$. Up to a subsequence, we may assume that $\left(u_{n}, v_{n}\right) \rightarrow\left(u_{\lambda}, v_{\lambda}\right)$ weakly in $H_{r}$ and strongly in $L^{q}\left(\mathbb{R}^{N}\right), q \in\left(2,2^{*}\right)$. Then $I_{\lambda}^{\prime}\left(u_{\lambda}, v_{\lambda}\right)=0$ and $\left(u_{\lambda}, v_{\lambda}\right)$ is a solution of (1.1). Moreover, by $\left(F_{1}\right)-\left(F_{2}\right)$ we have

$$
\lim _{n \rightarrow \infty} \int_{\mathbb{R}^{N}} f\left(u_{n}\right) u_{n}+g\left(v_{n}\right) v_{n} d x=\int_{\mathbb{R}^{N}} f\left(u_{\lambda}\right) u_{\lambda}+g\left(v_{\lambda}\right) v_{\lambda} d x .
$$

By $I_{\lambda}^{\prime}\left(u_{n}, v_{n}\right)\left(u_{n}, v_{n}\right)=o(1)$ we have

$$
\begin{aligned}
& \int_{\mathbb{R}^{N}}\left|\nabla u_{n}\right|^{2}+\left|\nabla v_{n}\right|^{2}+u_{n}^{2}+v_{n}^{2}-2 \lambda u_{n} v_{n} d x \\
= & \int_{\mathbb{R}^{N}} f\left(u_{n}\right) u_{n}+g\left(v_{n}\right) v_{n} d x+o(1) \\
= & \int_{\mathbb{R}^{N}} f\left(u_{\lambda}\right) u_{\lambda}+g\left(v_{\lambda}\right) v_{\lambda} d x+o(1) \\
= & \int_{\mathbb{R}^{N}}\left|\nabla u_{\lambda}\right|^{2}+\left|\nabla v_{\lambda}\right|^{2}+u_{\lambda}^{2}+v_{\lambda}^{2}-2 \lambda u_{\lambda} v_{\lambda} d x+o(1) .
\end{aligned}
$$

Therefore, $\left(u_{n}, v_{n}\right) \rightarrow\left(u_{\lambda}, v_{\lambda}\right)$ strongly in $H_{r}$, and so $\left(u_{\lambda}, v_{\lambda}\right) \in X^{\delta}$, which implies that $u_{\lambda} \not \equiv 0$ and $v_{\lambda} \not \equiv 0$. By Lemma 2.6 we have $u_{\lambda}>0$ and $v_{\lambda}>0$. Therefore, $\left(u_{\lambda}, v_{\lambda}\right)$ is a positive radially symmetric solution of (1.1). Then by the standard regularity theory, we see that $u_{\lambda}, v_{\lambda} \in C^{2}\left(\mathbb{R}^{N}\right)$. Moreover, $I_{\lambda}\left(u_{\lambda}, v_{\lambda}\right) \leq d_{\lambda}$.

Let $\lambda_{n} \in\left(0, \lambda_{0}\right), n \in \mathbb{N}$, be any sequence with $\lambda_{n} \rightarrow 0$ as $n \rightarrow \infty$. Then by repeating the proof of Lemma 2.3 and passing to a subsequence, $\left(u_{\lambda_{n}}, v_{\lambda_{n}}\right) \rightarrow(U, V)$ strongly in $H_{r}$ as $n \rightarrow \infty$, where $U \in S_{1}$ and $V \in S_{2}$. That is, $U$ is a positive radial ground state of (1.4), and $V$ is a positive radial ground state of (1.6) respectively. This completes the proof. 


\section{REFERENCES}

[1] Nail Akhmediev and Adrian Ankiewicz, Novel soliton states and bifurcation phenomena in nonlinear fiber couplers, Phys. Rev. Lett. 70 (1993), no. 16, 2395-2398, DOI 10.1103/PhysRevLett.70.2395. MR.1212560 (93k:35224)

[2] Antonio Ambrosetti, Remarks on some systems of nonlinear Schrödinger equations, J. Fixed Point Theory Appl. 4 (2008), no. 1, 35-46, DOI 10.1007/s11784-007-0035-4. MR 2447960 (2010c:34043)

[3] A. Ambrosetti, E. Colorado, and D. Ruiz, Multi-bump solitons to linearly coupled systems of nonlinear Schrödinger equations, Calc. Var. Partial Differential Equations 30 (2007), no. 1, 85-112, DOI 10.1007/s00526-006-0079-0. MR2333097 (2008m:35089)

[4] Antonio Ambrosetti, Giovanna Cerami, and David Ruiz, Solitons of linearly coupled systems of semilinear non-autonomous equations on $\mathbb{R}^{n}$, J. Funct. Anal. 254 (2008), no. 11, 28162845, DOI 10.1016/j.jfa.2007.11.013. MR2414222 (2009b:35069)

[5] Abbas Bahri and Yan Yan Li, On a min-max procedure for the existence of a positive solution for certain scalar field equations in $\mathbf{R}^{N}$, Rev. Mat. Iberoamericana 6 (1990), no. 1-2, 1-15, DOI 10.4171/RMI/92. MR 1086148 (92b:35054)

[6] Abbas Bahri and Pierre-Louis Lions, On the existence of a positive solution of semilinear elliptic equations in unbounded domains, Ann. Inst. H. Poincaré Anal. Non Linéaire 14 (1997), no. 3, 365-413 (English, with English and French summaries), DOI 10.1016/S02941449(97)80142-4. MR 1450954 (98k:35047)

[7] H. Berestycki and P.-L. Lions, Nonlinear scalar field equations. I. Existence of a ground state, Arch. Rational Mech. Anal. 82 (1983), no. 4, 313-345, DOI 10.1007/BF00250555. MR695535 (84h:35054a)

[8] Haïm Brezis and Elliott H. Lieb, Minimum action solutions of some vector field equations, Comm. Math. Phys. 96 (1984), no. 1, 97-113. MR765961 (86d:35045)

[9] Jaeyoung Byeon and Louis Jeanjean, Standing waves for nonlinear Schrödinger equations with a general nonlinearity, Arch. Ration. Mech. Anal. 185 (2007), no. 2, 185-200, DOI 10.1007/s00205-006-0019-3. MR2317788 (2008g:35049)

[10] Jaeyoung Byeon, Louis Jeanjean, and Mihai Mariş, Symmetry and monotonicity of least energy solutions, Calc. Var. Partial Differential Equations 36 (2009), no. 4, 481-492, DOI 10.1007/s00526-009-0238-1. MR 2558325 (2011f:35105)

[11] Giovanna Cerami, Some nonlinear elliptic problems in unbounded domains, Milan J. Math. 74 (2006), 47-77, DOI 10.1007/s00032-006-0059-z. MR2278729 (2007k:35128)

[12] Z. Chen and W. Zou, On coupled systems of Schrödinger equations, Adv. Differential Equations 16 (2011), no. 7-8, 775-800. MR2829504 (2012k:35109)

[13] David Gilbarg and Neil S. Trudinger, Elliptic partial differential equations of second order, 2nd ed., Grundlehren der Mathematischen Wissenschaften [Fundamental Principles of Mathematical Sciences], vol. 224, Springer-Verlag, Berlin, 1983. MR737190 (86c:35035)

[14] Louis Jeanjean and Kazunaga Tanaka, A remark on least energy solutions in $\mathbf{R}^{N}$, Proc. Amer. Math. Soc. 131 (2003), no. 8, 2399-2408 (electronic), DOI 10.1090/S0002-9939-0206821-1. MR1974637 (2004c:35127)

[15] Paul H. Rabinowitz, On a class of nonlinear Schrödinger equations, Z. Angew. Math. Phys. 43 (1992), no. 2, 270-291, DOI 10.1007/BF00946631. MR.1162728 (93h:35194)

[16] Michael Struwe, Variational methods. Applications to nonlinear partial differential equations and Hamiltonian systems, 2nd ed., Ergebnisse der Mathematik und ihrer Grenzgebiete (3) [Results in Mathematics and Related Areas (3)], vol. 34, Springer-Verlag, Berlin, 1996. MR1411681 (98f:49002)

Department of Mathematical Sciences, Tsinghua University, Beijing 100084, People's RePublic OF CHINA

E-mail address: chenzhijie1987@sina.com

Department of Mathematical Sciences, Tsinghua University, Beijing 100084, People's Republic OF CHinA

E-mail address: wzou@math.tsinghua.edu.cn 